\title{
The Predictive Value of Vessels Encapsulating Tumor Clusters in Treatment Optimization for Recurrent Early-stage Hepatocellular Carcinoma
}

\section{Zhi-Yuan Chen}

Hunan Provincial People 's Hospital

\section{Zhi-Xing Guo}

Sun Yat-sen University Cancer Center

Liang-He Lu

Sun Yat-sen University Cancer Center

Jie Mei

Sun Yat-sen University Cancer Center

Wen-Ping Lin

Sun Yat-sen University Cancer Center

Shao-Hua Li

Sun Yat-sen University Cancer Center

\section{Wei Wei}

Sun Yat-sen University Cancer Center

Rong-Ping Guo ( $\nabla$ guorp@sysucc.org.cn )

Sun Yat-sen University Cancer Center

\section{Research Article}

Keywords: Hepatocellular carcinoma, Vessels encapsulating tumor cluster, Radiofrequency ablation, Repeat hepatic resection

Posted Date: February 26th, 2021

DOI: https://doi.org/10.21203/rs.3.rs-228188/v1

License: (c) (i) This work is licensed under a Creative Commons Attribution 4.0 International License. Read Full License 


\section{Abstract}

Background: The predictive value of vessels encapsulating tumor clusters (VETC) in recurrent early-stage Hepatocellular Carcinoma (HCC) remains unclear. Therefore, the aim of the present study was to investigate the prognostic significance of VETC in patients with recurrent early-stage HCC after repeat hepatic resection (RHR) or radiofrequency ablation (RFA).

Methods: From December 2005 to December 2016, 138 patients receiving RHR and 188 patients receiving RFA were recruited. VETC was evaluated by immunohistochemical staining for CD34. The survival outcomes of patients with VETC pattern or not were investigated.

Results: There was no significant difference between the RHR and RFA groups in disease-free survival (DFS) or overall survival (OS) as determined by univariate analysis of the whole cohort. In the subgroup analysis of the VETC-positive cohort, the patients in the RHR group showed a longer median DFS time in contrast to those in the RFA group (15.0 vs 5.0 months, $P=0.001$ ). Similarly, the patients in the RHR group showed a longer median OS time in contrast to those in the RFA group (39.5 vs 19 months, $P=0.001$ ). In the VETC-negative cohort, no significant differences in DFS and OS rates between the RHR and RFA groups were observed $(P>0.05)$.

Conclusions: The results of our study suggested that RHR was relatively safe and superior to RFA in improving survival outcomes for recurrent early-stage HCC after initial hepatectomy. Furthermore, the VETC pattern may represent a reliable marker for selecting HCC patients who may benefit from RHR.

\section{Background}

Hepatocellular carcinoma (HCC) is characterized by high vascularization, rapid tumor progression and extremely poor outcome[1-3]. Surgical resection is commonly accepted as a curative treatment for HCC, however, the long-term outcomes are not yet satisfactory, as approximately $70 \%$ of patients experience recurrence within 5 years[4]. Approximately $30 \%$ of patients with recurrent $\mathrm{HCC}$ are diagnosed in the early stage, and consequently bear relatively favorable prognosis[5, 6]. However, guidelines for the management of recurrent early-stage HCC remain controversial and poorly defined.

Available management options for recurrent early-stage HCC are almost identical to those for primary HCC. Repeat hepatic resection (RHR) continues to be the conventional option for recurrent early-stage $\mathrm{HCC}$ with preserved liver function and residual liver volume, and progresses in surgical techniques have contributed to enhancing the safety of $\operatorname{RHR}[4,7,8]$. In addition, radiofrequency ablation (RFA), a minimally invasive option, has emerged as another alternative treatment modality for early-stage HCC[9, 10]. Several studies have previously recommended RHR when possible in the treatment of recurrent HCC[11-13]. However, conflicting data have shown that RFA, with relatively few complications, could achieve survival outcomes comparable to those of RHR for recurrent early-stage HCC[14-16]. Therefore, the optimal strategies for recurrent HCC remain unclear and controversial. 
We and other researchers have identified two microvessel types in $\mathrm{HCC}$ by their distinct morphologic features: capillary-like with small, scattered capillaries having no or narrow lumen and sinusoid-like that form a cobweb-like pattern and encapsulate tumor clusters, also named vessels encapsulating tumor clusters (VETC) $[17,18]$. The VETC pattern was found to be consistently and easily detectable in HCC and to indicate a poorer prognosis in patients after recurrence[19, 20]. Moreover, VETC pattern is acknowledged to be a predictor of sorafenib benefit in patients with HCC, especially those with VETC. VETC can be an indicator for guiding the treatment of patients with recurrent $\mathrm{HCC}[21]$. However, there are no studies regarding the prognostic value of the VETC pattern in recurrent early-stage HCC.

In this study, we used tissue specimens containing $326 \mathrm{HCC}$ samples from our center to compare the survival outcomes of recurrent early-stage HCC treated by RHR or RFA and explore whether VETC pattern could guide the identification of appropriate candidates for optimization treatment.

\section{Methods}

\section{Patients}

From December 2005 to December 2016, a total of 1206 HCC patients developed intrahepatic recurrence after initial R0 resection. Recurrence was diagnosed either by histologic findings or by the noninvasive criteria used by the American Association for the Study of Liver Diseases. Among the patients, 144 (11.9\%) were amenable to RHR, and 203 (16.8\%) received RFA. The inclusion criteria were as follows: (a) age between 18 and 75 years; (b) first intrahepatic recurrence of HCC after curative hepatectomy; (c) single lesion $\leq 3 \mathrm{~cm}$ or $2-3$ lesions $\leq 3 \mathrm{~cm}$ recurrent HCC; (d) no radiologic evidence of macroscopic vascular invasion or extrahepatic metastasis; (e) RHR or RFA performed as the initial treatment for recurrent HCC; and (f) Child-Pugh class A or B. Thus, six patients in the RHR cohort were excluded, namely, four patients who received palliative repeated resection and two patients with other malignancies. Fifteen patients in the RFA cohort were excluded, including five patients older than 75 years, four patients with extrahepatic metastasis, and six patients whose samples were not sufficient for immunohistochemistry staining. Finally, totally 326 patients were recruited into the current study, including 138 patients receiving RHR and 188 patients receiving RFA as the first treatment for recurrent early-stage HCC. The study got the approval of the Institutional Review Board of Sun Yat-sen University Cancer Center and conducted in line with the ethical guidelines of the Declaration of Helsinki.

\section{Treatment strategy}

Treatment for recurrent HCC at early-stage was performed as previously described[22]. RHR was assigned when there was the possibility for the complete removal of all tumors while retaining a sufficient liver remnant, with an expected remnant liver volume of no less than $250 \mathrm{ml} / \mathrm{m}^{2}$, as evaluated by our multidisciplinary team. Resection was avoided if patients had gross ascites, severe portal hypertension, or an inadequate liver remnant. Reasons for assigning RFA instead of RHR included psychological resistance to invasive treatment, refusal of general anesthesia, and an insufficient liver remnant. 


\section{Immunohistochemistry staining}

The sensitive streptavidin-biotinylated horseradish peroxidase complex system (Catalyzed Signal Amplification System, DAKO, Carpinteria, CA) was utilized for the immunohistochemistry staining for CD34 based on the manufacturer's instructions. Formalin-fixed and paraffin-embedded sections were dewaxed in xylene and rehydrated in gradient ethanol. Afterwards, endogenous peroxidase activity was blocked by $3 \%$ hydrogen peroxide for $10 \mathrm{~min}$. Antigen retrieval was performed by microwave pretreatment in $100 \mathrm{~W}$ citrate buffers for $5 \mathrm{~min}$ and $30 \mathrm{~W}$ for $25 \mathrm{~min}$. Then, the sections were incubated with mouse anti-human CD34 monoclonal antibody (mAb; working dilution 1:200, QBEnd10, DAKO) at $4^{\circ} \mathrm{C}$ overnight. Following washing by TBS with $0.1 \%$ Tween 20 , the sections were incubated with biotinylated rabbit antimouse secondary antibody for $30 \mathrm{~min}$, followed by TBS washing. The sections were then incubated with streptavidin-biotin complex for $15 \mathrm{~min}$. All the sections were counterstained with hematoxylin.

\section{Outcome measures}

Immunohistochemical staining was independently evaluated using the same diagnostic criteria by two pathologists who were blinded to the patient data. In case of discrepancy, the specimens were referred to a third observer, and the majority decision was considered final. Using a Leica inverted research microscope (Leica Microsystems, Wetzlar, Germany), the slides were examined under 100x magnification to identify the highest vascular density area within the tumor, and five representative fields were observed at a higher magnification of 200x. Microvascular invasion (MVI) is defined as microscopic tumor invasion in the central hepatic vein, the portal, or large capsular vessels[23, 24]. Tumor differentiation was histologically graded according to the criteria proposed by WHO classification of Tumors of the Digestive System (2010 version).

\section{Statistical analysis}

Disease-free survival (DFS) and overall survival (OS) rates were calculated with the life table method, and the survival time was calculated from the day of surgery to the day of death or the most recent follow-up visit. The patient survival curves were compared using the Kaplan-Meier method and analyzed using the log-rank test. Chi-square tests and Spearman's rank tests were adopted to evaluate the univariate correlation between the biological parameters and clinicopathological variables, as well as the recurrence. The relevant prognostic factors were identified using multivariate Cox proportional hazards models. Statistical Package for the Social Sciences software (SPSS version 19.0, IBM, Armonk, NY, USA) was utilized for data analysis. The $P$ value was obtained from a two-tailed test, and $P<0.05$ meant a statistical difference.

\section{Results}

\section{Subject characteristics}


The baseline characteristics of patients with recurrent early-stage HCC were summarized in Table 1. In all, 80 patients were VETC-positive and 145 patients were VETC-negative. We also found 101 patients with mixed VETC and capillary-like microvessels in the tumor tissue simultaneously (Figure 1). Lastly, 119 patients were classified as VETC-positive (defined as staining of VETC $>50 \%$ ), and 207 patients were classified as VETC-negative (defined as staining of capillary-like microvessels $>50 \%$ ) (Figure 2).

Among VETC-positive HCC patients, 50 patients underwent RHR and 69 patients underwent RFA; among VETC-negative HCC patients, 88 patients received RHR and 119 patients received RFA. The proportions of VETC cases were similar in cohorts 1 and 2 (42.0\% vs 42.5\%) and between the RHR and RFA groups. Notably, the VETC pattern was more frequently observed with the occurrence of MVI and early recurrence. In the subgroups of patients with VETC-positive or VETC-negative lesions, little significant difference could be observed between the RHR and RFA groups for any of the baseline characteristics.

\section{Survival analysis in the overall cohort}

The median duration of follow-up was 39 months (range, 2-118 months). At the time of censoring, 256 $(78.5 \%)$ of 326 patients had recurred, and $153(46.9 \%)$ had died of tumor progression. The 1-, 3-, and 5year DFS rates for the VETC-positive group and VETC-negative group were $39.5 \%, 17.6 \%$, and $8.4 \%$ and $50.7 \%, 29.5 \%$, and $18.8 \%$, respectively. The VETC-negative group had a longer median DFS time compared with the VETC-positive group (6.9 vs 12.0 months, $P=0.011$ ). Similarly, the $1-, 3-$, and 5-year OS rates for the VETC-positive group and VETC-negative group were $74.8 \%, 44.5 \%$, and $19.3 \%$ and $87.4 \%$, $58.9 \%$, and $33.8 \%$, respectively. The VETC-negative group had a longer median OS time compared with the VETC-positive group (27 vs 46 months, $P=0.002$ ) (Table 2).

Univariate analysis exhibited that time to recurrence, VETC and microvascular invasion were found to be significant risk factors affecting DFS and OS. There was little significant difference between the RHR and RFA groups for DFS and OS. By multivariate analysis, time to recurrence $(P<0.001)$, tumor multiplicity $(P=0.008)$, Child-Pugh score at recurrence $(P<0.001)$, microvascular invasion $(P<0.001)$ and VETC pattern $(P=0.015)$ were recognized as independent predictors of DFS. Time to recurrence $(P=0.002)$, Child-Pugh score at recurrence $(P=0.004)$, microvascular invasion $(P=0.019)$ and VETC pattern $(P=0.025)$ were identified as independent predictors of OS (Table 3 ).

First, the survival benefit of treatment for recurrent early-stage HCC in the VETC-positive and VETCnegative groups was evaluated. In the VETC-positive cohort, the 1-, 3-, 5-year DFS rates were $58.0 \%$, $30.0 \%$, and $14.0 \%$ for the RHR group and $26.1 \%, 8.7 \%$, and $4.3 \%$ for the RFA group, respectively. The RHR group had a longer median DFS time compared to the RFA group (15.0 vs 5.0 months, $P=0.001$ ). Similarly, the 1-, 3-, and 5-year OS rates for RHR group and RFA group were $82.0 \%, 54.0 \%$, and $28.0 \%$ and $69.6 \%, 37.7 \%$, and $13.0 \%$, respectively $(P=0.008)$. The RHR group had a longer median OS time compared to the RFA group (39.5 vs 19 months, $P=0.001$ ) (Figure 3). In contrast, in the VETC-negative cohort (Figure 4 ) and the entire cohort (Figure 5), there was no significant difference in DFS and OS rates between the RHR and RFA groups $(P>0.05)$ (Figure 4). 


\section{Characteristics after treatment}

Treatment-related complications were summarized in Supplementary table 1. Complications were reported according to the Clavien-Dindo classification. No treatment-related mortality was reported in this study, and the incidence of major complications in the RHR group did not differ significantly from that of the RFA group. Nevertheless, the delay in the RHR group resulted in a notably longer hospital stay (6.7 vs 4.1 day, $P<0.001)$ and higher total hospital charges $(52,645$ vs $27,949 \mathrm{RMB}, P<0.001)$.

\section{Discussion}

Because of the high incidence of intrahepatic recurrence, which affects the prognosis of HCC patients, the available treatment options are not that different from those for primary tumors. Many recent articles have reported the feasibility, safety, and efficacy of RHR or RFA for intrahepatic HCC recurrence. However, there is no consensus on the most appropriate choice for HCC recurrence with regard to RHR or RFA. Our study demonstrated that RHR was relatively safe and superior to RFA in improving survival outcomes for recurrence of early-stage HCC for our whole cohort. Moreover, we first explored the VETC pattern to identify the patient subgroup mostly likely to benefit from this treatment. The result showed that HCC patients who were VETC-positive could benefit from RHR, while there was no significant difference between RHR and RFA in the VETC-negative cohort.

Indeed, following surgical resection of the primary tumors, intensive screening was usually applied using AFP levels and CT or MRI, and the recurring tumors were usually detected in the early stage, with less than $3 \mathrm{~cm}$ tumor size[25]. Our study showed that approximately $30 \%$ of recurrent HCCs were smaller than $3 \mathrm{~cm}$, which was more similar to the real situation in the recurrence of tumors. As expected, recurrent early-stage $\mathrm{HCC}$ with a smaller tumor size was closely related to a higher rate of complete tumor elimination after RFA and a greater safety resection margin with fewer resections of tumor-free liver parenchyma in RHR, which in turn produces better prognosis. However, different from the prognosis of first treatment in early-stage HCC, several studies have shown that $70-80 \%$ of patients developed a second recurrence with either RHR or RFA[26-28]. Our study found that $78.5 \%$ of patients had recurred, and $46.9 \%$ had died of tumor progression after the second treatment. This may be because recurrent $\mathrm{HCC}$, with a higher degree of malignancy, more easily leads to a second recurrence after treatment.

The debate about whether resection or ablation should be first-line therapy for early-stage HCC has intensified over the years, and there has also been controversy about treatment optimization for recurrent early-stage HCC. Several studies revealed similar survival outcomes between RHR and RFA in recurrent small tumors[22, 29, 30]. Compared with RHR, RFA is a highly selective, targeted thermal treatment technique to conserve tumor-free liver parenchyma and cause a minimum of damage to the small or cirrhotic liver remnant. Unlike surgery, RFA can be performed under conscious sedation and has a shorter hospital day, making it more cost-effective than surgical resection. In our study, the median total hospital stay duration was shorter and median total charges for patients who underwent RFA were significantly lower than those for patients who underwent RHR. Therefore, RFA is usually the first choice for patients 
with early-stage HCC, whether primary or recurrent. Our study showed that $57.7 \%$ of patients had RFA and $42.3 \%$ of patients had RHR. No significant difference in survival outcomes between RFA and RHR in the whole cohort could be observed.

The VETC pattern has been identified as an effective predictor of survival in patients with HCC after resection, and it can be easily identified and distinguished from capillary vessels by using IHC staining for a single endothelial marker, CD34[17]. In our study, we found that the VETC pattern was correlated not only with recurrence after first resection but also with second recurrence. Furthermore, we found that the VETC-positive patients in the RHR group had longer median DFS and OS rates than those in the RFA group, while there was no significant difference in DFS or OS rates between the RHR and RFA groups among the VETC-negative cohort. VETC is closely associated with tumor micrometastases and venous thrombus in the tumor margins. We postulate that RFA cannot achieve sufficient ablation in hepatic tumors with potential adjacent venous invasion or microscopic lesion remnants. In contrast, RHR can usually achieve a relatively satisfactory margin of normal liver tissue if possible. Another explanation may be that RFA is inferior to surgery in local tumor control. Recurrence more than 2 years after initial resection is now commonly considered to attributed by multicentric carcinogenesis due to hepatitis or cirrhosis. As the latest advancement of hepatic surgical techniques, anatomic resection is well developed and is performed more actively for hepatic tumors, which can remove the tumor and the entire Couinaud segment potentially containing the adjacent venous invasion or microscopic lesions simultaneously[31].

Several limitations existed in our study. First, since this was a retrospective study, selection bias may have influenced the results. Second, treatment selection for recurrent HCC was decided by our multidisciplinary team and not randomly assigned. For HCC patients who had a major resection, when recurrence was present and located deep within the liver or in a patient with insufficient liver function reserve, RFA was usually the first choice as it causes less damage, making patients more likely to benefit from treatment.

\section{Conclusions}

In conclusion, the results of our study showed that RHR was relatively safe and superior to RFA in improving survival outcomes for recurrent early-stage HCC after initial hepatectomy. Additionally, the VETC pattern may represent a reliable marker for selecting HCC patients who may benefit from RHR; however, large-scale studies are required to confirm these findings.

\section{List Of Abbreviations}

VETC: vessels encapsulating tumor clusters; HCC: hepatocellular carcinoma; RHR: repeat hepatic resection; RFA: radiofrequency ablation; DFS: disease-free survival; OS: overall survival.

\section{Declarations}


Acknowledgements

Not applicable.

\section{Author contributions}

ZYC, ZXG, and LHL contributed equally to this work; WW and RPG also contributed equally to this work. ZYC, WW, and RPG conceived and designed the experiments; ZYC, ZXG, LHL, and WPL collected the data; ZXG and JM analyzed the data; ZYC wrote the manuscript; SHL revised the manuscript. All authors have read and approved the final version of the manuscript.

\section{Funding}

This study was supported by Hunan Provincial People's Hosptial RenShu Foundation (RS201805), the National Natural Science Foundation of China (No. 81871985) and Natural Science Foundation of Guangdong Province (No. 2017A030310203).

\section{Availability of data and materials}

The dataset analyzed during the current study are available from the corresponding author on reasonable request.

\section{Ethics approval and consent to participate}

All study participants provided informed consent. The study got the approval of the Institutional Review Board of Sun Yat-sen University Cancer Center and conducted in line with the ethical guidelines of the Declaration of Helsinki.

\section{Consent for publication}

Not applicable.

\section{Competing interests}


None reported.

\section{References}

1. Bray F, Ferlay J, Soerjomataram I, Siegel RL, Torre LA, Jemal A: Global cancer statistics 2018: GLOBOCAN estimates of incidence and mortality worldwide for 36 cancers in 185 countries. CA: a cancer journal for clinicians 2018, 68(6):394-424.

2. Arzumanyan A, Reis HM, Feitelson MA: Pathogenic mechanisms in HBV- and HCV-associated hepatocellular carcinoma. Nature reviews Cancer 2013, 13(2):123-135.

3. Feng RM, Zong YN, Cao SM, Xu RH: Current cancer situation in China: good or bad news from the 2018 Global Cancer Statistics? Cancer Commun (Lond) 2019, 39(1):22.

4. Itamoto T, Nakahara H, Amano H, Kohashi T, Ohdan H, Tashiro H, Asahara T: Repeat hepatectomy for recurrent hepatocellular carcinoma. Surgery 2007, 141(5):589-597.

5. Imamura H, Matsuyama Y, Tanaka E, Ohkubo T, Hasegawa K, Miyagawa S, Sugawara Y, Minagawa $M$, Takayama T, Kawasaki $S$ et al: Risk factors contributing to early and late phase intrahepatic recurrence of hepatocellular carcinoma after hepatectomy. Journal of hepatology 2003, 38(2):200207.

6. Lee MW, Lim HK: Management of sub-centimeter recurrent hepatocellular carcinoma after curative treatment: Current status and future. World journal of gastroenterology 2018, 24(46):5215-5222.

7. Zhou Y, Sui C, Li B, Yin Z, Tan Y, Yang J, Liu Z: Repeat hepatectomy for recurrent hepatocellular carcinoma: a local experience and a systematic review. World journal of surgical oncology 2010, 8:55.

8. Utsunomiya T, Okamoto M, Kameyama T, Matsuyama A, Yamamoto M, Fujiwara M, Mori M, Aimitsu $\mathrm{S}$, Ishida T: Impact of obesity on the surgical outcome following repeat hepatic resection in Japanese patients with recurrent hepatocellular carcinoma. World journal of gastroenterology 2008, 14(10):1553-1558.

9. Yang W, Yan K, Goldberg SN, Ahmed M, Lee JC, Wu W, Zhang ZY, Wang S, Chen MH: Ten-year survival of hepatocellular carcinoma patients undergoing radiofrequency ablation as a first-line treatment. World journal of gastroenterology 2016, 22(10):2993-3005.

10. Huang GL, Liu M, Zhang XE, Liu BX, Xu M, Lin MX, Kuang M, Lu MD, Xie XY: Multiple-Electrode Switching-Based Radiofrequency Ablation vs. Conventional Radiofrequency Ablation for Single EarlyStage Hepatocellular Carcinoma Ranging From 2 to 5 Cm. Frontiers in oncology 2020, 10:1150.

11. Jiang YQ, Wang ZX, Deng YN, Yang Y, Wang GY, Chen GH: Efficacy of Hepatic Resection vs. Radiofrequency Ablation for Patients With Very-Early-Stage or Early-Stage Hepatocellular Carcinoma: A Population-Based Study With Stratification by Age and Tumor Size. Frontiers in oncology 2019, 9:113.

12. Peng Y, Liu F, Wei Y, Li B: Outcomes of laparoscopic repeat liver resection for recurrent liver cancer: $A$ system review and meta-analysis. Medicine 2019, 98(41):e17533. 
13. Lacaze L, Scotte M: Surgical treatment of intra hepatic recurrence of hepatocellular carcinoma. World journal of hepatology 2015, 7(13):1755-1760.

14. Lee GC, Ferrone CR, Vagefi PA, Uppot RN, Tanabe KK, Lillemoe KD, Blaszkowsky LS, Qadan M: Surgical resection versus ablation for early-stage hepatocellular carcinoma: A retrospective cohort analysis. American journal of surgery 2019, 218(1):157-163.

15. Tohme S, Geller DA, Cardinal JS, Chen HW, Packiam V, Reddy S, Steel J, Marsh JW, Tsung A: Radiofrequency ablation compared to resection in early-stage hepatocellular carcinoma. HPB : the official journal of the International Hepato Pancreato Biliary Association 2013, 15(3):210-217.

16. Wang Y, Luo Q, Li Y, Deng S, Wei S, Li X: Radiofrequency ablation versus hepatic resection for small hepatocellular carcinomas: a meta-analysis of randomized and nonrandomized controlled trials. PloS one 2014, 9(1):e84484.

17. Chen. ZY, Wei. W, Guo. ZX, Lin. JR, Shi. M, Guo RP: Morphologic classification of microvessels in hepatocellular carcinoma is associated with the prognosis after resection. Journal of Gastroenterology and Hepatology 2011, 26(5):866-874.

18. Ding. T, Xu. J, Zhang. Y, Guo. RP, Wu. WC, Zhang. SD, Qian. CN, Zheng L: Endothelium-coated tumor clusters are associated with poor prognosis and micrometastasis of hepatocellular carcinoma after resection. Cancer 2011, 117(21):4878-4889.

19. Yamashita YI, Baba H: Vessels encapsulating tumor clusters in hepatocellular carcinoma: a unique and valuable pathological imaging. Hepatobiliary surgery and nutrition 2020, 9(4):484-487.

20. He C, Zhou Z, Jiang H, Yin Z, Meng S, Zhang J, Huang P, Xu K, Bian L, Xiao Z et al: EpithelialMesenchymal Transition is Superior to Vessels-Encapsulate Tumor Cluster in Promoting Metastasis of Hepatocellular Carcinoma: a Morphological Evidence. Journal of Cancer 2017, 8(1):39-47.

21. Jian-Hong Fang LX, Li-Ru Shang, Chu-Zhi Pan, Jin Ding, Yun-Qiang Tang, Hui Liu, Chu-Xing Liu, JiaLin Zheng, Yao-Jun Zhang, Zhong-Guo Zhou, Jing Xu, Limin Zheng, Min-Shan Chen, Shi-Mei Zhuang: Vessels That Encapsulate Tumor Clusters (VETC) Pattern Is a Predictor of Sorafenib Benefit in Patients with Hepatocellular Carcinoma. Hepatology 2019, 70(3):824-839.

22. Lu LH, Mei J, Kan A, Ling YH, Li SH, Wei W, Chen MS, Zhang YF, Guo RP: Treatment optimization for recurrent hepatocellular carcinoma: Repeat hepatic resection versus radiofrequency ablation. Cancer medicine 2020, 9(9):2997-3005.

23. Wei W, Jian PE, Li SH, Guo ZX, Zhang YF, Ling YH, Lin XJ, Xu L, Shi M, Zheng L et al: Adjuvant transcatheter arterial chemoembolization after curative resection for hepatocellular carcinoma patients with solitary tumor and microvascular invasion: a randomized clinical trial of efficacy and safety. Cancer Commun (Lond) 2018, 38(1):61.

24. Vauthey JN, Lauwers GY, Esnaola NF, Do KA, Belghiti J, Mirza N, Curley SA, Ellis LM, Regimbeau JM, Rashid A et al: Simplified staging for hepatocellular carcinoma. Journal of clinical oncology : official journal of the American Society of Clinical Oncology 2002, 20(6):1527-1536.

25. Wen T, Jin C, Facciorusso A, Donadon M, Han HS, Mao Y, Dai C, Cheng S, Zhang B, Peng B et al: Multidisciplinary management of recurrent and metastatic hepatocellular carcinoma after resection: 
an international expert consensus. Hepatobiliary surgery and nutrition 2018, 7(5):353-371.

26. Wang HL, Mo DC, Zhong JH, Ma L, Wu FX, Xiang BD, Li LQ: Systematic review of treatment strategy for recurrent hepatocellular carcinoma: Salvage liver transplantation or curative locoregional therapy. Medicine 2019, 98(8):e14498.

27. Cai H, Kong W, Zhou T, Qiu Y: Radiofrequency ablation versus reresection in treating recurrent hepatocellular carcinoma: a meta-analysis. Medicine 2014, 93(22):e122.

28. Na BG, Kim JM, Oh DK, Lee KW, Kang TW, Choi GS, Lee MW, Kwon CHD, Lim HC, Joh JW: Clinical outcomes of laparoscopic radiofrequency ablation of single primary or recurrent hepatocellular carcinoma ( $</=3 \mathrm{~cm})$. Annals of surgical treatment and research 2017, 92(5):355-360.

29. He ZX, Xiang P, Gong JP, Cheng NS, Zhang W: Radiofrequency ablation versus resection for Barcelona clinic liver cancer very early/early stage hepatocellular carcinoma: a systematic review. Therapeutics and clinical risk management 2016, 12:295-303.

30. Duan C, Liu M, Zhang Z, Ma K, Bie P: Radiofrequency ablation versus hepatic resection for the treatment of early-stage hepatocellular carcinoma meeting Milan criteria: a systematic review and meta-analysis. World journal of surgical oncology 2013, 11(1):190.

31. Shin S, Kim TS, Lee JW, Ahn KS, Kim YH, Kang KJ: Is the anatomical resection necessary for single hepatocellular carcinoma smaller than $3 \mathrm{~cm}$ ?: single-center experience of liver resection for a small HCC. Annals of hepato-biliary-pancreatic surgery 2018, 22(4):326-334.

\section{Tables}

Table 1 Patent characteristics at the time of recurrence 


\begin{tabular}{|c|c|c|c|c|c|c|}
\hline & $\operatorname{VETC}(+)$ & & & VETC (-) & & \\
\hline Variables & $\mathrm{RHR}$ & RFA & $P$ & $\mathrm{RHR}$ & RFA & $P$ \\
\hline Age, y & $49.5 \pm 10.9$ & $48.9 \pm 10.7$ & 0.822 & $51.51 \pm 10.3$ & $49.4 \pm 11.2$ & 0.498 \\
\hline Sex & & & 1.000 & & & 0.647 \\
\hline Male & 47 & 64 & & 78 & 108 & \\
\hline Female & 3 & 5 & & 10 & 11 & \\
\hline HBsAg & & & 0.427 & & & 0.201 \\
\hline Positive & 41 & 61 & & 76 & 94 & \\
\hline Negative & 9 & 8 & & 12 & 25 & \\
\hline Background liver & & & 0.708 & & & 0.137 \\
\hline Normal & 19 & 29 & & 34 & 34 & \\
\hline Cirrhosis & 31 & 40 & & 54 & 85 & \\
\hline Histological grade & & & 0.850 & & & 0.776 \\
\hline Well differentiated & 21 & 27 & & 38 & 48 & \\
\hline Poorly differentiated & 29 & 42 & & 50 & 71 & \\
\hline Microvascular invasion & & & 0.136 & & & 0.472 \\
\hline Present & 18 & 35 & & 14 & 24 & \\
\hline Absent & 32 & 34 & & 74 & 95 & \\
\hline ALB at recurrence, $g / L$ & $41.9 \pm 6.6$ & $42.0 \pm 3.3$ & 0.154 & $42.6 \pm 5.8$ & $42.4 \pm 7.7$ & 0.488 \\
\hline $\begin{array}{l}\text { TBIL at recurrence, } \\
\text { umol/L }\end{array}$ & $15.5 \pm 4.7$ & $14.7 \pm 4.9$ & 0.922 & $15.5 \pm 5.6$ & $15.2 \pm 5.9$ & 0.147 \\
\hline HGB at recurrence, $\mathrm{g} / \mathrm{L}$ & $143.6 \pm 17.8$ & $143.9 \pm 16.8$ & 0.421 & $144.3 \pm 22.4$ & $142.7 \pm 24.0$ & 0.777 \\
\hline $\begin{array}{l}\text { AFP at recurrence, } \\
\mathrm{ng} / \mathrm{ml}\end{array}$ & & & 0.838 & & & 0.884 \\
\hline$>20$ & 35 & 50 & & 56 & 77 & \\
\hline$\leq 20$ & 15 & 19 & & 32 & 42 & \\
\hline $\begin{array}{l}\text { Child-Pugh score at } \\
\text { recurrence }\end{array}$ & & & 0.488 & & & 0.363 \\
\hline 5 & 42 & 54 & & 73 & 94 & \\
\hline 6 & 8 & 15 & & 13 & 25 & \\
\hline Tumor size at & $2.4 \pm 0.6$ & $2.2 \pm 0.5$ & 0.472 & $2.4 \pm 0.5$ & $2.3 \pm 0.4$ & 0.630 \\
\hline
\end{tabular}


recurrence, $\mathrm{cm}$

Tumor multiplicity at

0.451

0.158 recurrence

\begin{tabular}{lcccc} 
Solitary & 44 & 57 & 75 & 91 \\
Multiple & 6 & 12 & 13 & 28 \\
\hline $\begin{array}{l}\text { Time to recurrence } \\
<1 \text { year }\end{array}$ & 23 & 27 & 0.192 & \\
$\geq 1$ year & 41 & 28 & 44 & 54 \\
\hline
\end{tabular}

Table 2 Comparison of DFS rate and OS rate in entire series (Kaplan-Meier method) 


\begin{tabular}{|c|c|c|c|c|c|}
\hline Variables & $\mathrm{n}$ & DFS & $P$ & OS & $P$ \\
\hline HBsAg & & & 0.935 & & 0.932 \\
\hline Positive & 272 & $10.0 \pm 0.9$ & & $38.5 \pm 13.2$ & \\
\hline Negative & 54 & $14.1 \pm 4.8$ & & $39.0 \pm 16.2$ & \\
\hline Background liver & & & 0.609 & & 0.674 \\
\hline Normal & 116 & $11.0 \pm 1.4$ & & $39 \pm 13.3$ & \\
\hline Cirrhosis & 210 & $10.0 \pm 1.6$ & & $39.0 \pm 14.8$ & \\
\hline Histological grade & & & 0.028 & & 0.648 \\
\hline Well differentiated & 192 & $10.8 \pm 2.2$ & & $39.0 \pm 13.7$ & \\
\hline Poorly differentiated & 134 & $10.0 \pm 1.3$ & & $38.5 \pm 14.4$ & \\
\hline Microvascular invasion & & & $<0.001$ & & 0.002 \\
\hline Present & 90 & $13.8 \pm 1.8$ & & $46.0 \pm 14.1$ & \\
\hline Absent & 236 & $5.7 \pm 0.9$ & & $27.0 \pm 13.5$ & \\
\hline AFP at recurrence, $\mathrm{ng} / \mathrm{ml}$ & & & 0.204 & & 0.123 \\
\hline$>20$ & 108 & $11.0 \pm 3.4$ & & $43.5 \pm 14.6$ & \\
\hline$\leq 20$ & 218 & $10.0 \pm 1.2$ & & $36.5 \pm 9.4$ & \\
\hline Child-Pugh score at recurrence & & & 0.148 & & 0.195 \\
\hline 5 & 146 & $11.0 \pm 1.5$ & & $34.5 \pm 11.5$ & \\
\hline 6 & 180 & $10.0 \pm 1.6$ & & $44.0 \pm 13.5$ & \\
\hline Tumor multiplicity at recurrence & & & 0.094 & & 0.686 \\
\hline Solitary & 267 & $11 \pm 1.5$ & & $39 \pm 17.9$ & \\
\hline Multiple & 59 & $7 \pm 2.3$ & & $39 \pm 14.3$ & \\
\hline Time to recurrence & & & $<0.001$ & & $<0.001$ \\
\hline$<1$ year & 144 & $7.0 \pm 0.9$ & & $33 \pm 8.3$ & \\
\hline$\geq 1$ year & 182 & $18.0 \pm 2.7$ & & $50.5 \pm 15.0$ & \\
\hline VETC & & & 0.011 & & 0.002 \\
\hline Positive & 119 & $6.9 \pm 1.4$ & & $27.0 \pm 10.1$ & \\
\hline Negative & 207 & $12.0 \pm 1.8$ & & $46.0 \pm 13.5$ & \\
\hline Treatment allocation & & & 0.114 & & 0.259 \\
\hline
\end{tabular}




$\begin{array}{llll}\text { RHR } & 138 & 11.8 \pm 3.3 & 42.0 \pm 12.6 \\ \text { RFA } & 188 & 10.0 \pm 1.1 & 37.5 \pm 10.4\end{array}$

Table 3 Multivariate analysis of the risk factors related to DFS and OS of recurrent early-stage HCC

\begin{tabular}{|c|c|c|c|c|c|c|}
\hline & \multicolumn{3}{|l|}{ DFS } & \multicolumn{3}{|l|}{ OS } \\
\hline & $P$ & $\mathrm{HR}$ & $95 \% \mathrm{Cl}$ & $P$ & $\mathrm{HR}$ & $\begin{array}{l}95 \% \\
\mathrm{Cl}\end{array}$ \\
\hline HBsAg (positive/negative) & 0.912 & 1.019 & $\begin{array}{l}0.725- \\
1.433\end{array}$ & 0.779 & 1.065 & $\begin{array}{l}0.685- \\
1.656\end{array}$ \\
\hline Cirrhosis (present/absent) & 0.627 & 0.937 & $\begin{array}{l}0.719- \\
1.220\end{array}$ & 0.326 & 1.186 & $\begin{array}{l}0.844- \\
1.667\end{array}$ \\
\hline $\begin{array}{l}\text { Tumor multiplicity at recurrence } \\
\text { (solitary/multiple) }\end{array}$ & 0.008 & 1.554 & $\begin{array}{l}1.124- \\
2.148\end{array}$ & 0.514 & 1.150 & $\begin{array}{l}0.755- \\
1.752\end{array}$ \\
\hline AFP at recurrence, $\mathrm{ng} / \mathrm{mL}(\leq 20 />20)$ & 0.461 & 1.107 & $\begin{array}{l}0.845- \\
1.450\end{array}$ & 0.193 & 1.262 & $\begin{array}{l}0.889- \\
1.793\end{array}$ \\
\hline Child-Pugh score at recurrence $(5 / 6)$ & 0.000 & 0.466 & $\begin{array}{l}0.338- \\
0.641\end{array}$ & 0.004 & 0.541 & $\begin{array}{l}0.357- \\
0.820\end{array}$ \\
\hline $\begin{array}{l}\text { Histological grade (well/poorly } \\
\text { differentiated) }\end{array}$ & 0.292 & 1.147 & $\begin{array}{l}0.889- \\
1.479\end{array}$ & 0.354 & 0.855 & $\begin{array}{l}0.614- \\
1.191\end{array}$ \\
\hline Time to recurrence ( $<1$ year/ $\geq 1$ year) & 0.000 & 0.556 & $\begin{array}{l}0.431- \\
0.716\end{array}$ & 0.002 & 0.601 & $\begin{array}{l}0.435- \\
0.832\end{array}$ \\
\hline Microvascular invasion (present/absent) & 0.000 & 2.997 & $\begin{array}{l}2.103- \\
4.273\end{array}$ & 0.019 & 1.739 & $\begin{array}{l}1.097- \\
2.757\end{array}$ \\
\hline Treatment allocation (RHR/RFA) & 0.228 & 1.170 & $\begin{array}{l}0.907- \\
1.510\end{array}$ & 0.459 & 1.133 & $\begin{array}{l}0.815- \\
1.575\end{array}$ \\
\hline VETC (positive/negative) & 0.015 & 1.454 & $\begin{array}{l}0.854- \\
1.948\end{array}$ & 0.025 & 1.486 & $\begin{array}{l}1.050- \\
2.102\end{array}$ \\
\hline
\end{tabular}

\section{Figures}




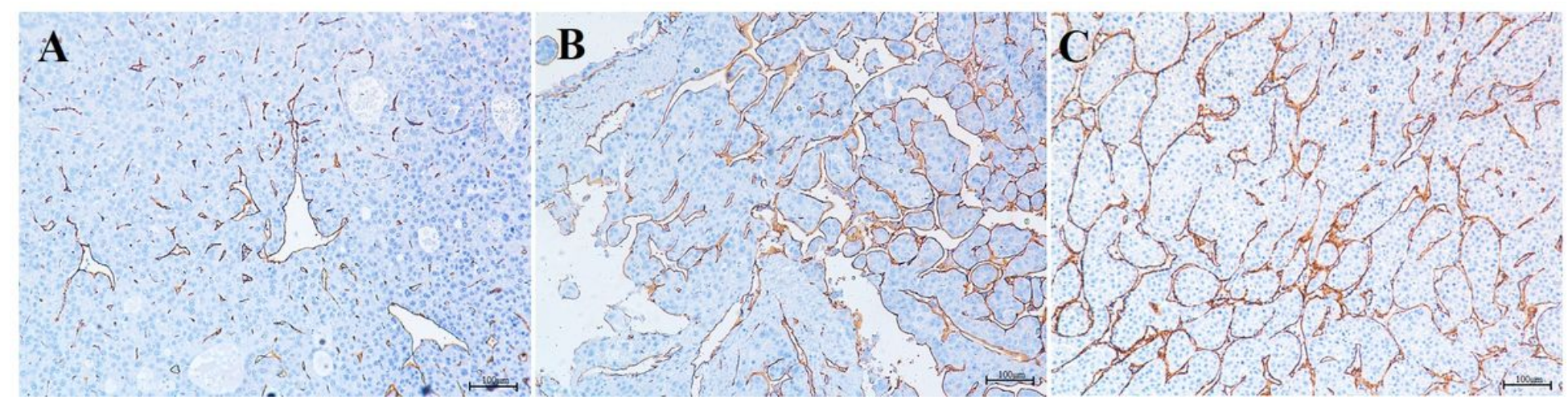

Figure 1

IHC staining for human CD34 was performed to detect vascular patterns in human HCC tissues. (A) VETC-positive: sinusoid-like that form a cobweb-like pattern and encapsulate tumor clusters; (B) VETCnegative: capillary-like microvessel; (C) mixed VETC and capillary-like microvessel. IHC: immunohistochemical staining, HCC: hepatocellular carcinoma, VETC: vessels encapsulating tumor clusters.

\section{Patients with $\mathrm{HCC}$ developed recurrence after RO curative hepatectomy $(\mathrm{n}=1206)$}

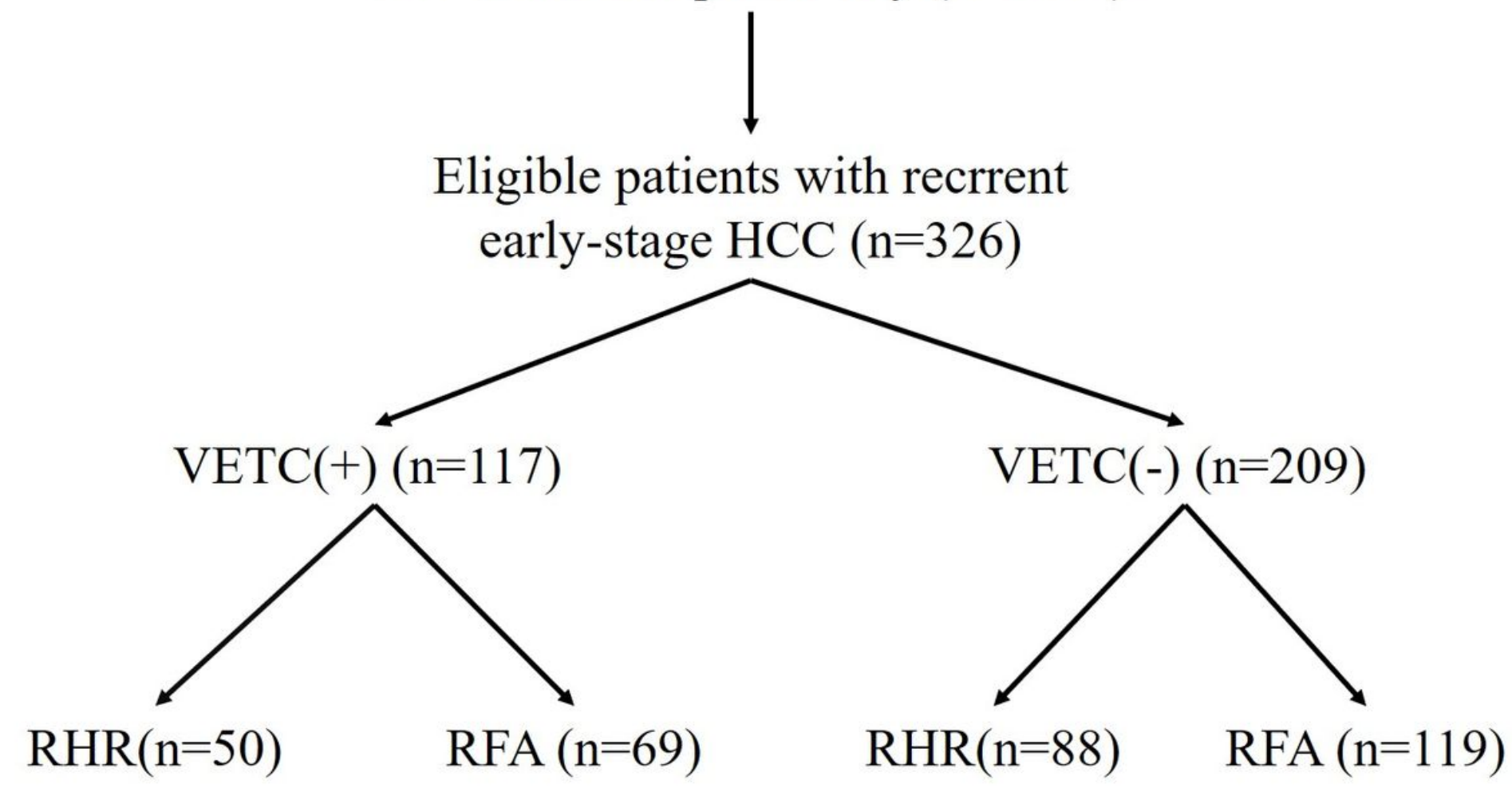

Figure 2

Flow chart of the study protocol. RHR: repeat hepatic resection, RFA: radiofrequency ablation. 

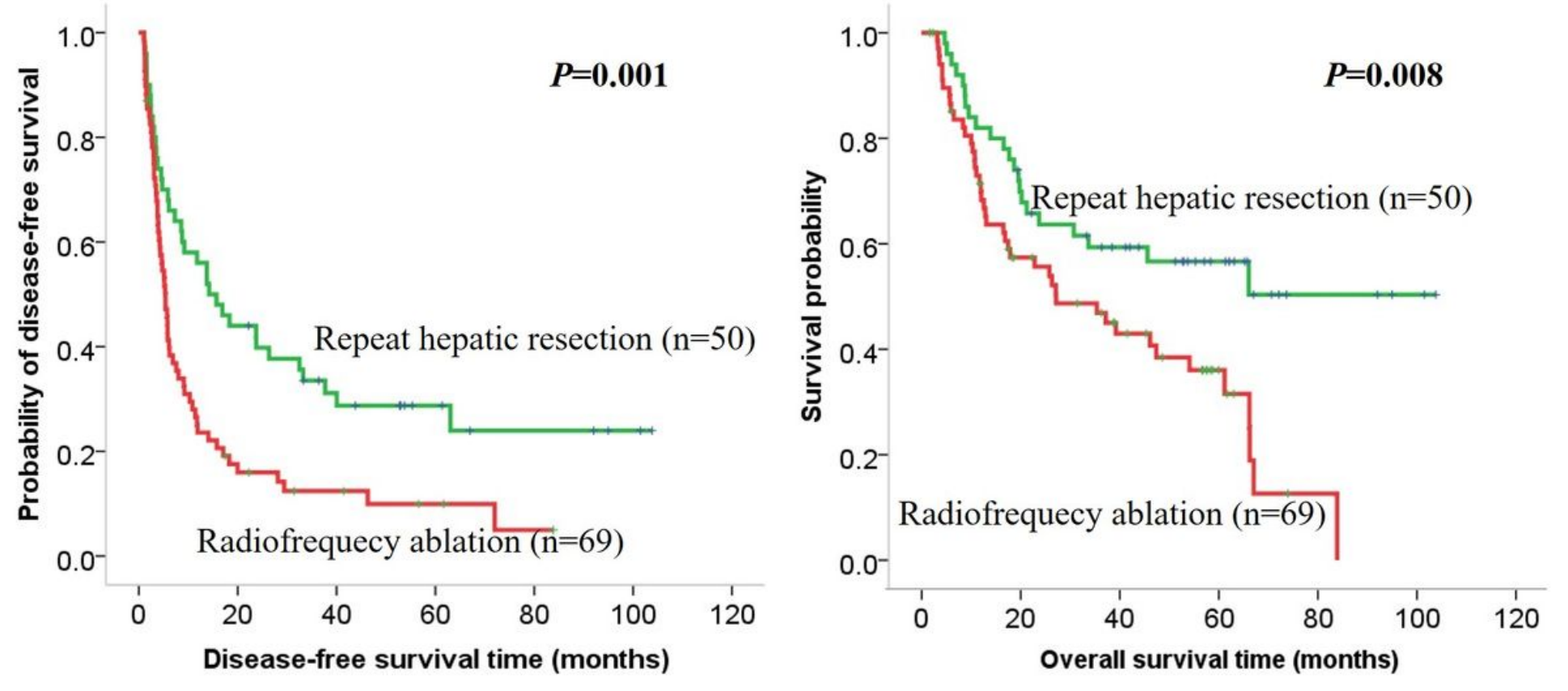

Figure 3

Kaplan-Meier curves of survival outcomes of recurrent early-stage HCC. Kaplan-Meier curves of (A) DFS and (B) OS in the VETC-positive cohort. DFS: disease-free survival, OS: overall survival.
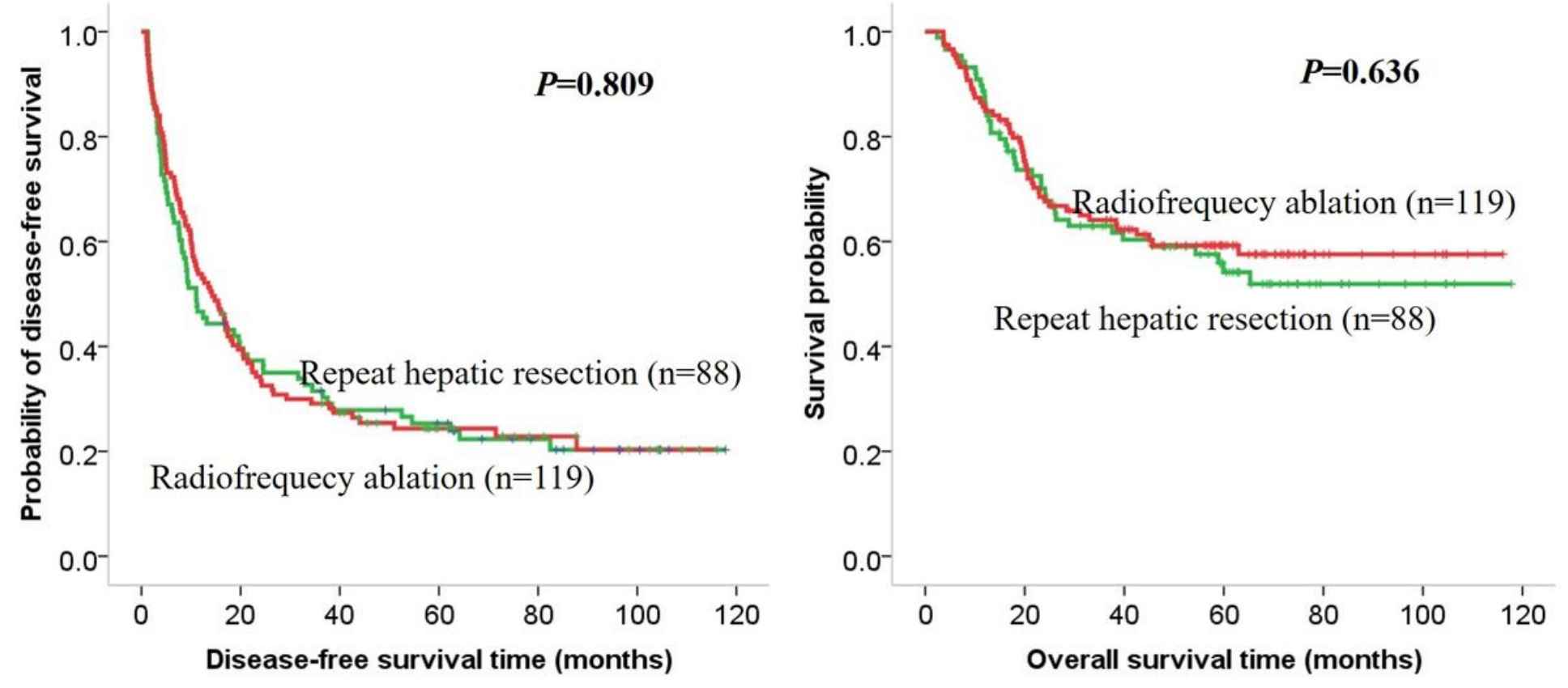

\section{Figure 4}

Kaplan-Meier curves of survival outcomes of recurrent early-stage HCC. Kaplan-Meier curves of (A) DFS and (B) OS in the VETC-negative cohort. 

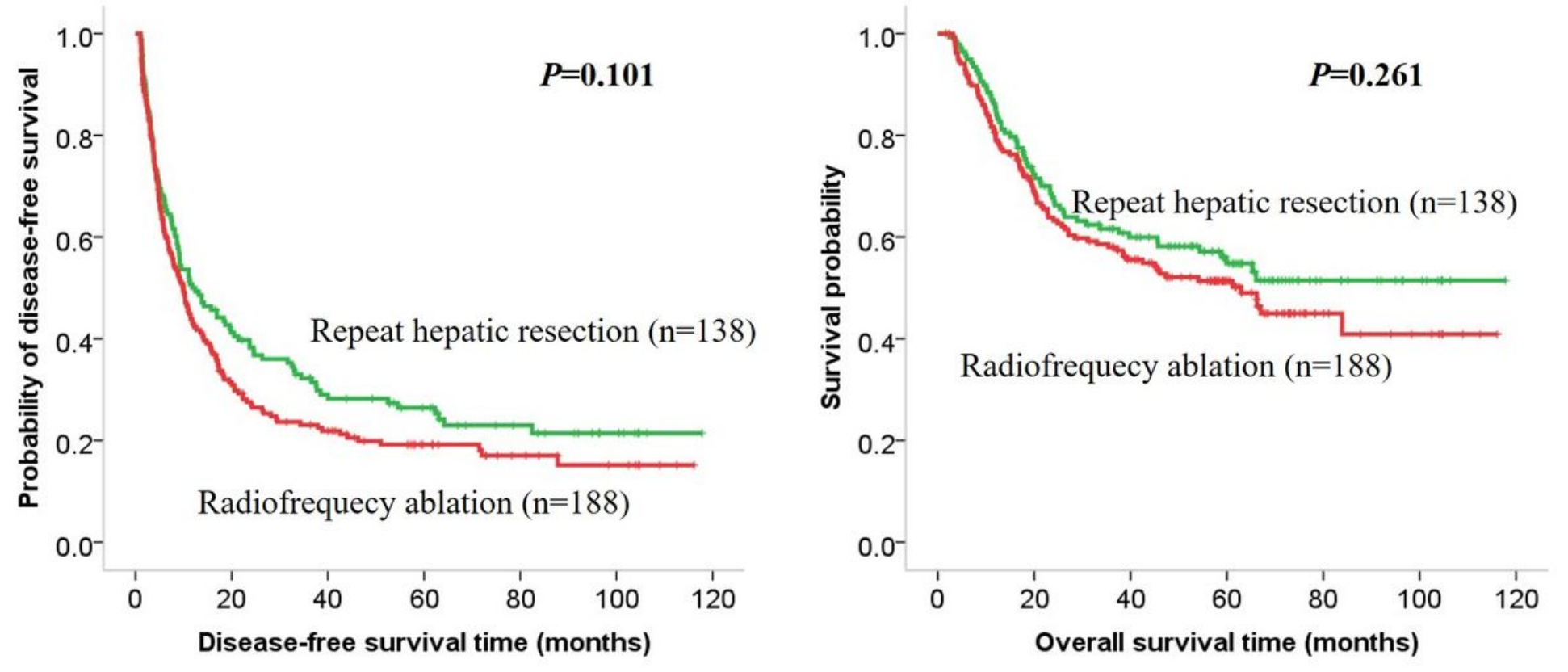

\section{Figure 5}

Kaplan-Meier curves of survival outcomes of recurrent early-stage HCC. Kaplan-Meier curves of (A) DFS and (B) OS in the whole cohort.

\section{Supplementary Files}

This is a list of supplementary files associated with this preprint. Click to download.

- Supplementarytable1.docx 